\title{
DAC-less Amplifier-less Generation and Transmission of QAM Signals Using Sub-Volt Silicon-organic Hybrid Modulators
}

\author{
S. Wolf, M. Lauermann, P. C. Schindler, G. Ronniger, K. Geistert, R. Palmer, S. Koeber, W. Bogaerts, \\ Senior Member, IEEE J. Leuthold, Fellow, IEEE, W. Freude, Senior Member, IEEE, and C. Koos, \\ Member, IEEE
}

\begin{abstract}
We demonstrate generation and transmission of optical signals by directly interfacing highly efficient siliconorganic hybrid (SOH) modulators to binary output ports of an FPGA. Using an SOH Mach-Zehnder modulator (MZM) and an SOH IQ modulator we generate on-off-keying (OOK) and binary phase shift keying (BPSK) signals as well as quadrature phase shift keying (QPSK) and 16-state quadrature amplitude modulation (16QAM) formats. Peak-to-peak voltages amount to only $0.27 \mathrm{~V}_{\mathrm{pp}}$ for driving the MZM and $0.41 \mathrm{~V}_{\mathrm{pp}}$ for the IQ modulator. Neither digital-to-analog converters (DAC) nor drive amplifiers are required, and the $\mathrm{RF}$ energy consumption in the modulator amounts to record-low $18 \mathrm{fJ} / \mathrm{bit}$ for 16QAM signaling.
\end{abstract}

Index Terms - Electrooptic modulation, Optical transmitters, Phase shift keying, Photonic integrated circuits, Quadrature amplitude modulation, Silicon photonics

\section{INTRODUCTION}

$\mathrm{H}$ IGH-capacity optical interconnects are key to overcome transmission bottlenecks in informationprocessing systems. In this context, intimate co-integration and direct interfacing of photonic and electronic circuitry [1] is indispensable to realize low-cost transceiver modules with high throughput, low power consumption, and the capability of using wavelength division multiplexing. At the same time, optimized spectral efficiency becomes increasingly important

Manuscript received November 03, 2014. This work was supported by the European Research Council (ERC Starting Grant 'EnTeraPIC', 280145), the EU projects PHOXTROT, BigPipes and SOFI, the Alfried Krupp von Bohlen und Halbach Foundation, the International Research School for Teratronics (HIRST), the Karlsruhe School of Optics and Photonics (KSOP), and the Karlsruhe Nano-Micro Facility (KNMF).

S. Wolf, M. Lauermann, G. Ronniger, K. Geistert W. Freude, and C. Koos are with the Institute of Photonics and Quantum Electronics (IPQ), Karlsruhe Institute of Technology (KIT), 76131 Karlsruhe, Germany (email: s.wolf@kit.edu; christian.koos@kit.edu).

P. C. Schindler was with KIT and is now with Infinera Corporation, Sunnyvale, CA, USA (pschindler@infinera.com). R. Palmer was with KIT and is now with Coriant $\mathrm{GmbH}$, Munich, Germany. S. Koeber was with KIT and is now with University of Cologne, Chemistry Department, Cologne, Germany. J. Leuthold was with KIT and is now with the Swiss Federal Institute of Technology (ETH), Zurich, Switzerland.

W. Bogarts is with Photonics Research Group, Ghent University - imec, Department of Information Technology, Gent, Belgium and is now also with Luceda Photonics, Dendermonde, Belgium.

C. Koos is also with the Institute of Microstructure Technology (IMT) at KIT, 76344 Eggenstein-Leopoldshafen, Germany.

(C) () 2015 IEEE. Personal use of this material is permitted. Permission from IEEE must be obtained for all other uses, in any current or future media, including reprinting/republishing this material for advertising or promotional purposes, creating new collective works, for resale or redistribution to servers or lists, or reuse of any copyrighted component of this work in other works. not only for telecommunications but also for optical interconnects in data centers, where space for fiber bundles is a precious resource and where installed fibers represent the most costly part of the communication system. This calls for higher-order modulation formats [2] such as quadrature phaseshift keying (QPSK) or $M$-ary quadrature amplitude modulation ( $M$-QAM). However, the associated technical complexity, cost and power consumption of today's photonicelectronic interfaces is still prohibitive for optical interconnects: The generation of higher-order modulation formats currently relies either on high-speed digital-to-analog converters (DAC) and linear electrical amplifiers or other dedicated electronic circuitry to generate the drive signals for electro-optic modulators. The energy consumption of DACs and amplifiers is considerable: Operating a 3-bit power DAC [3] at a peak-to-peak voltage of $3.2 \mathrm{~V}$ and a symbol rate of $42 \mathrm{GBd}$ requires a total power of $2 \mathrm{~W}$, which corresponds to an energy consumption of $2 \mathrm{~W} /(42 \mathrm{GBd} \times 3 \mathrm{bit})=$ $16 \mathrm{pJ} /$ bit. This is already in the order of the total package power dissipation of small form-factor pluggables (SFP) or $120 \mathrm{Gbit} / \mathrm{s}$ active optical cables (AOC) that are based on directly modulated vertical-cavity surface-emitting lasers (VCSEL). Power-efficient DAC-less amplifier-less transmitters are therefore indispensable for realizing highspeed optical interconnects that exploit co-integration of photonics with digital CMOS electronics for generating advanced modulation formats. In the context of this paper, the terms "DAC-less" and "amplifier-less" describe a scheme in which binary output ports of a digital CMOS circuit are connected to an optical modulator solely via a passive electrical network that generates multi-level drive signals without the use of further active elements.

For conventional on-off-keying (OOK), amplifier-less interfaces between the outputs of field-programmable gate arrays (FPGA) and silicon-photonic micro-ring modulators were demonstrated at line rates of $2.5 \mathrm{Gbit} / \mathrm{s}$ and drive voltages of $1.2 \mathrm{~V}_{\mathrm{pp}}$ [4]. The comparatively low drive voltage was enabled by the resonant behavior of the micro-ring modulator, which allows overcoming the rather low efficiency of depletion-type modulators. However, this concept cannot be easily transferred to the generation of higher-order modulation formats where the phase needs to be controlled independently 
of the amplitude. Moreover, the scheme requires to carefully match the ring resonance to the carrier wavelength by, e.g., heaters, which increase the power consumption considerably. Regarding advanced modulation formats, DAC-less generation of 16QAM optical signals was proposed and demonstrated by using serial or parallel configurations of multiple Mach-Zehnder modulators (MZM), each operated with binary drive signals [5]-[7]. However, this approach still requires electrical amplifiers to drive the various MZMs with peak-to-peak voltages of typically more than $2 \mathrm{~V}[7]$. More recently, generation of a 16QAM optical signal from binary electrical drive signals was demonstrated using IQ modulators that consist of MZMs with segmented phase shifters [8]. However, in this experiment, an additional electrical driver circuit was needed to interface the analog drive inputs of the modulator to the digital outputs of the data source. This leads to an additional electrical power consumption of approximately $1 \mathrm{~W}$, corresponding to an energy consumption of $18 \mathrm{pJ} /$ bit at a data rate of $56 \mathrm{Gbit} / \mathrm{s}$.

In this paper, we report on proof-of-concept experiments demonstrating that non-resonant and yet highly-efficient silicon-based modulators that can be operated directly with binary sub-Volt output signals of standard field-programmable gate arrays (FPGA) without the need for digital-to-analog converters or drive amplifiers [9], [10]. These devices are realized on the silicon-organic hybrid ( $\mathrm{SOH}$ ) platform [11][13], which enables generation of a wide variety of modulation formats at lowest energy consumption [14]-[17]. We demonstrate the viability of the approach in a series of experiments: Using SOH MZM and IQ modulators, we show generation and transmission of on-off-keying (OOK) and binary phase shift keying (BPSK) signals at $10 \mathrm{Gbit} / \mathrm{s}$ as well as QPSK and 16QAM signaling at $13 \mathrm{GBd}$. For the 16 QAM experiment, we use peak-to-peak drive voltages of only $0.41 \mathrm{~V}_{\mathrm{pp}}$, leading to an ultra-low RF energy consumption of only $18 \mathrm{fJ} /$ bit in the modulator - far below that of comparable all-silicon devices. We believe that DAC-less and amplifierless interfaces between digital circuitry and highly efficient $\mathrm{SOH}$ modulators can enable a novel class of highly scalable optical interconnects with unprecedented energy efficiency.

The paper is organized as follows: Section II explains specifics of the SOH technology platform and describes the structure of the modulators. Experiments with an FPGAdriven Mach-Zehnder modulator using $10 \mathrm{Gbit} / \mathrm{s}$ OOK and BPSK formats are presented in Section III. In Section IV, the DAC-less generation of QPSK and 16QAM signals is demonstrated, and data transmission over a distance of up to $100 \mathrm{~km}$ with a data rate of up to $52 \mathrm{Gbit} / \mathrm{s}$ is shown. Finally, we discuss the RF energy requirements of our modulators in Section V.

\section{Silicon ORganic Hybrid (SOH) EleCtro-Optic MODULATORS}

Our silicon-organic hybrid ( $\mathrm{SOH}$ ) modulators combine silicon-on-insulator (SOI) slot waveguides with organic electro-optic (EO) claddings [13], [18]. The optical slot waveguide has two silicon "rails" [13], Fig. 1(a). The space (a)

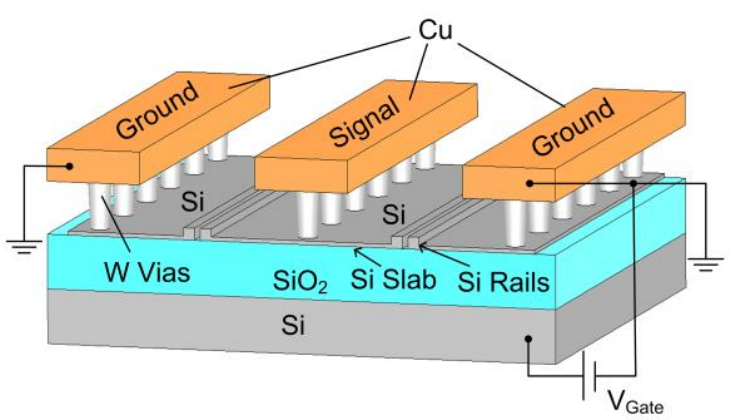

(b)

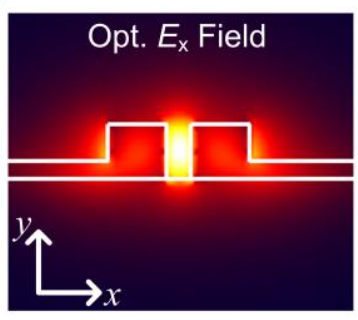

(c)

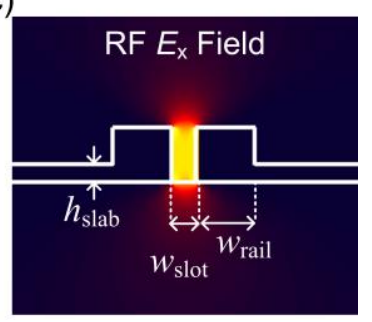

Fig. 1. Schematic of an SOH MZM. The device consists of two phase modulators driven in push-pull operation by a single coplanar transmission line (ground-signal-ground, GSG). The GSG transmission line is made of copper $(\mathrm{Cu})$ strips that are connected to the $\mathrm{Si}$ rails of the slot waveguide $900 \mathrm{~nm}$ high tungsten $(\mathrm{W})$ vias and thin $n$-doped silicon slabs. The silicon slot waveguide ( $\left.\mathrm{w}_{\text {Slot }}=120 \mathrm{~nm}, \mathrm{w}_{\text {Rail }}=240 \mathrm{~nm}, \mathrm{~h}_{\text {Rail }}=220 \mathrm{~nm}\right)$ is coated with the electro-optic material SEO100 (not shown). (b) $E_{\mathrm{x}}$ component of the optical field in the slot waveguide. (c) $E_{\mathrm{x}}$ component of the electrical RF drive signal. The optical mode and the modulation field are both confined to the slot, resulting in strong interaction and hence in efficient modulation.

between them is filled with an electro-optic organic material. For quasi-TE polarization, the dominant field component $E_{x}$ in the slot is strongly enhanced due to the discontinuity of the normal electric field component at the interface to the silicon rails. If a voltage is applied across the slot, the refractive index changes and the phase of the optical wave is modulated. Two such phase shifter sections form the arms of an SOH MachZehnder interferometer as illustrated in Fig. 1(a). By controlling the phase shift in both arms in a push-pull configuration, the light amplitude is modulated without introducing a residual phase shift. For modulation at microwave frequencies, copper electrodes in form of a ground-signal-ground (GSG) transmission line are added. The metal electrodes are connected to the slot by $900 \mathrm{~nm}$ high tungsten vias and thin $n$-doped silicon slabs, so that the modulating voltage drops mainly across the narrow slot of each phase shifter section. A cross-section of an SOH phase shifter together with the optical and RF field is depicted in Fig. 1(b) and (c), respectively. The optical field strongly overlaps with the modulating RF field in the slot, leading to highly-efficient electro-optic interaction and hence low drive voltage requirements.

The underlying SOI structures were fabricated in IMEC's active silicon photonics platform, which includes three etch steps for the silicon waveguides as well as implants and silicidation for the electrically contacted devices. The EO material is deposited on the pre-processed device by spincoating and fills the slot homogeneously. A separate step [12] is necessary for poling the electro-optic cladding: At an elevated temperature, a poling voltage across the (floating) 


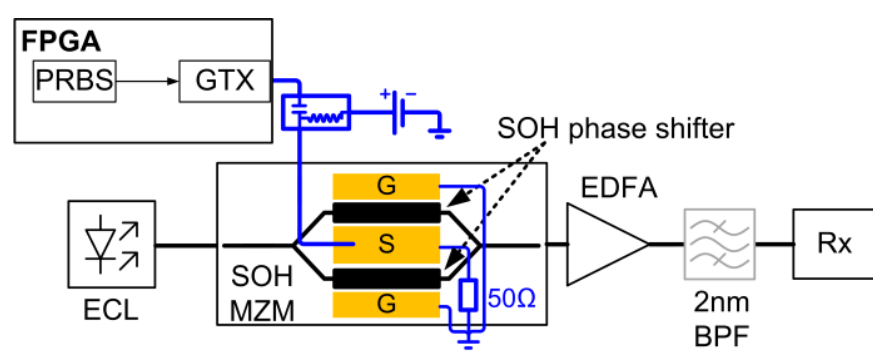

Fig. 2. Experimental setup for directly driving an $\mathrm{SOH}$ modulator with the GTX interfaces of a Virtex-7 FPGA. A pseudo-random binary sequence (PRBS) at the GTX output ports is applied to the Mach-Zehnder modulator (MZM) without further amplification. The MZM consists of two $1 \mathrm{~mm}$ long SOH phase shifters that are driven by a ground-signal-ground (GSG) transmission line. Drive signal and bias voltage are combined in a bias-tee. The optical carrier from an external cavity laser (ECL) is modulated in the SOH MZM, and an erbium-doped fiber amplifier (EDFA) compensates the insertion loss before the signal is fed to the receiver (Rx). For coherent detection, an optical modulation analyzer (OMA) is used, whereas direct detection relies on a single photodiode. For direct detection, a $2 \mathrm{~nm}$ bandpass filter (BPF) suppresses ASE noise of the EDFA.

ground electrodes aligns the EO chromophores in the same direction for both slots. If a modulating RF voltage is applied to the signal electrode, the associated field is oriented in opposite directions in the two arms of the MZM, leading to phase shifts of the same magnitude but opposite sign and hence to efficient push-pull operation [13]. As an EO cladding material we use SEO100 from Soluxra, LLC. The material is specified for a high electro-optic coefficient of $110 \mathrm{pm} / \mathrm{V}$ and operating temperatures of up to $85^{\circ} \mathrm{C}$.

The electrical bandwidth of the modulators is limited by RC low-pass characteristics arising from the capacitance of the slot which has to be charged and discharged via the resistive silicon slabs [18]. To increase the modulation bandwidth, a static gate field of $0.1 \mathrm{~V} / \mathrm{nm}$ is applied between the bulk silicon and the SOI device layer. The gate field leads to an electron accumulation layer and to a higher conductivity of the silicon slabs and hence to an increased bandwidth of the devices [18]-[20]. The gate voltage does not have any appreciable effect on the power consumption: The associated current flow is of the order of a few nanoamperes, corresponding to an energy consumption of a few aJ/bit, which can safely be neglected. In future device generations, the gate voltage may be reduced significantly by depositing a thin silicon layer on top of the silicon slabs [18] or even be omitted by employing an improved doping profile which increases the conductivity of the silicon slabs.

The $\pi$-voltage-length product of the modulators is below $0.8 \mathrm{Vmm}$. The $\pi$-voltages have been measured at bias voltages of $2 \mathrm{~V}$ or more in order to prevent screening of the applied fields by free charges in the cladding, which may occur at zero or small bias. Due to the low mobility of the free charges, this effect is only observable for low frequencies and does not impede high-frequency operation [12].

\section{BINARY DRIVEN SOH MACH-ZEHNDER MODULATOR WITHOUT ELECTRICAL AMPLIFICATION}

In a first set of experiments, we use a single electrical drive signal which is fed to an MZM to generate OOK and BPSK signals. The experimental setup is depicted in Fig. 2. Light (a) $10 \mathrm{GBd}$ electrical drive signal

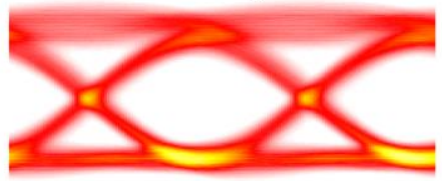

(c)

(b) Optical signal after direct detection
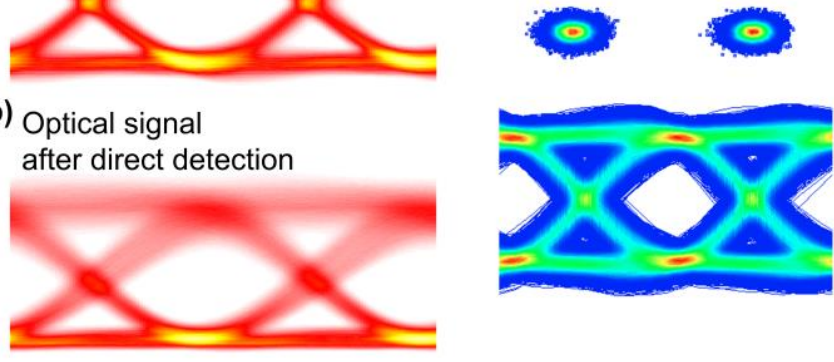

Fig. 3. Electrical drive signals and corresponding optical signals for OOK and BPSK at $10 \mathrm{Gbit} / \mathrm{s}$. (a) Eye diagram of electrical output of GTX transmitter. (b) Eye diagram of the optical OOK signal after direct detection by a photodiode. The quality factor $\mathrm{Q}=6.7$ indicates error-free detection. (c) Constellation diagram (top) and eye diagram (bottom) of optical BPSK signal obtained after coherent reception. An error-vector magnitude (EVM) of $13.7 \%$ indicating error-free signaling [27] (BER $<10^{-9}$ ) is measured.

from an external-cavity laser (ECL) is coupled to a $1 \mathrm{~mm}$ long SOH MZM. The device features a $\pi$-voltage of $0.74 \mathrm{~V}$ at DC, measured at a bias voltage of around $3.5 \mathrm{~V}$ to prevent screening effects [12]. At the output of the modulator chip, the optical signal is amplified by an erbium-doped fiber amplifier (EDFA) to compensate for the insertion loss of the MZM chip. The total fiber-to-fiber insertion loss of our current devices amounts to approximately $20 \mathrm{~dB}$ and is dominated by the fiber-chip coupling loss of non-optimized grating couplers of about $6 \mathrm{~dB}$ per interface. The on-chip loss of the MZM amounts to approximately $8 \mathrm{~dB}$ for maximum transmission of the modulator and can be decomposed into losses of passive components $(<1 \mathrm{~dB})$, free-carrier absorption within the doped silicon waveguides of $1.5 \mathrm{~dB}$, and scattering loss of $5.5 \mathrm{~dB}$ due to sidewall roughness of the slot waveguide. These losses can be reduced considerably in future device generations. It has been shown that the roughness-induced propagation loss of strip-loaded slot waveguides can be diminished to $0.2 \mathrm{~dB} / \mathrm{mm}$ by improved fabrication procedures and by using asymmetric cross sections [21]. Similarly, optimization of the doping profiles can lead to carrier-induced propagation losses of less than $1 \mathrm{~dB} / \mathrm{mm}$ such that the total insertion loss of the $1 \mathrm{~mm}$-long slot-waveguide section amounts to less than $1.2 \mathrm{~dB}$. Each phase shifter of the MZM consists of a slot waveguide and two rib-to-slot converters, each of which contributes another $0.02 \mathrm{~dB}$ of insertion loss [22]. Power splitting and combining is accomplished by a pair of multimode interference couplers (MMI), for which the insertion losses can be as low as $0.2 \mathrm{~dB}$ per coupler [23]. This would amount to total on-chip insertion losses of less than $1.7 \mathrm{~dB}$ for an SOH MZM with $1 \mathrm{~mm}$-long phase shifter sections. For packaged devices, we may assume fiber-to-chip coupling losses of less than $1.7 \mathrm{~dB}$ per interface, enabled by optimized grating couplers [24] or by photonic wire bonds [25], and propagation loss of less than $0.2 \mathrm{~dB}$ in few millimeters of lowloss access waveguides [26]. We hence expect that fiber-tofiber insertion losses can be reduced to less than $5.3 \mathrm{~dB}$ for an 


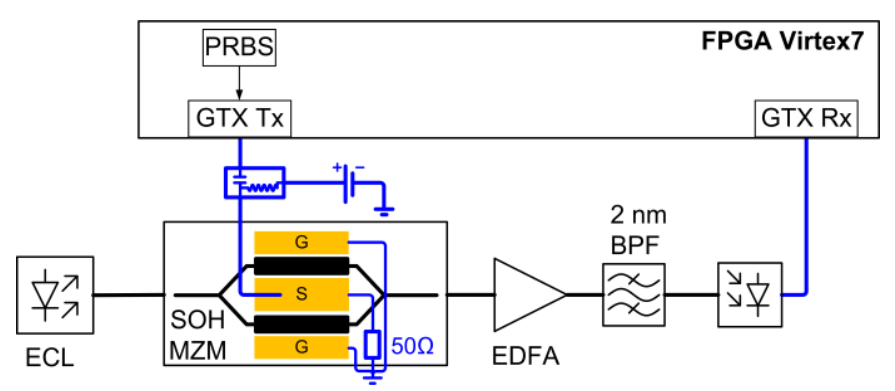

Fig. 4. Experimental setup for OOK transmission experiment: Laser light from an ECL is coupled to the SOH modulator that is directly driven from the GTX Tx output of the FPGA. The signal is amplified by an erbium-doped fiber amplifier (EDFA), and a band-pass filter with a $2 \mathrm{~nm}$ passband is used to suppress amplified spontaneous emission (ASE) noise. A photodiode is used for direct detection of the signal. The electrical signal extracted from the photodiode and sent to a GTX Rx port of the same FPGA without further electrical amplification. Data analysis showed BER of $6.6 \times 10^{-6}$.

MZM. These estimations are entirely based on already demonstrated structures; further improvements will be possible as better components become available. As a consequence, the EDFA can be omitted for data transmission using future device generations. In this context, the power handling capability of the device appears as an important parameter, which is directly linked to the photostability of the organic material. This aspect is subject to ongoing investigations. In our experiment, we operated each of the $\mathrm{SOH}$ phase shifters with optical powers of more than $5 \mathrm{dBm}$ over several hours without observing any photo-induced deterioration of the device performance. This power was not limited by the photostability of the EO material, but by the output power of the transmitter laser and the insertion loss of the device. We expect that systematic investigations of the photostability will exhibit damage thresholds that are significantly larger. Still, the currently used $5 \mathrm{dBm}$ of on-chip power per phase shifter would correspond to a total output power of more than $7.6 \mathrm{dBm}$, taking into account the insertion losses of optimized MMI power combiners and assuming full transmission of the MZM. This should enable EDFA-less transmission in future devices.

After amplification, the signal is fed to the optical receiver. The receivers are chosen differently depending on the modulation format: A coherent receiver ( $\mathrm{Rx})$ using another ECL as a local oscillator is used for coherent BPSK demodulation, whereas direct detection on a fast photodiode is performed for OOK signals. In the case of direct detection, a $2 \mathrm{~nm}$ bandpass filter (BPF) suppresses amplified spontaneous emission (ASE) from the optical amplifier.

For the experiment, we use a Xilinx XC7VX485T FPGA on a VC707 evaluation board. A pseudo-random binary sequence (PRBS) of length $2^{31}-1$ is generated on the FPGA and fed to a single GTX transmitter (Tx) for OOK and BPSK modulation. GTX transceivers are specified to operate at a maximum of $0.5 \mathrm{~V}_{\mathrm{pp}}$ for single-ended operation in the low-frequency limit. In our case, we measure a swing of about $310 \mathrm{mV}_{\mathrm{pp}}$ from the electric eye diagram at the transmitter output. The Xilinx GTX interfaces are neither designed to drive photonic devices nor do they provide a flat frequency response. The pronounced low-pass characteristic was compensated by an internal digital 3-tap finite-impulse response (FIR) filter. Using the FIR filter

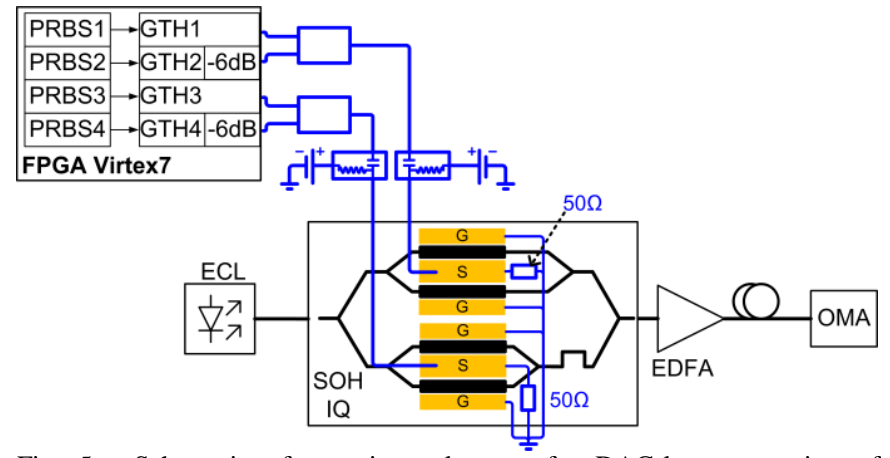

Fig. 5. Schematic of experimental setup for DAC-less generation of 16QAM. The silicon-organic hybrid (SOH) IQ modulator consists of two $1.5 \mathrm{~mm}$ long Mach-Zehnder modulators (MZM). Each MZM is driven by a ground-signal-ground (GSG) transmission line contacted via a bias-tee and a microwave probe (not shown) and terminated with a $50 \Omega$ resistor. An intentional optical path length imbalance in the parent MZM allows to adjust an optical $\pi / 2$ phase shift between I and Q via wavelength tuning. To generate the four-level drive signal for each MZM, the PRBS signals from two GTH ports with independent output voltages differing by $6 \mathrm{~dB}$ are combined in a power combiner. The resulting 4-level signal is applied to the modulator electrodes. An external cavity laser (ECL) is used as an optical source, and an erbium-doped fiber amplifier (EDFA) compensates the optical insertion loss of the device before the signal is fed to the optical modulation analyzer (OMA).

configured to high-pass behavior results in a nicely open eye diagram of the electrical signal, Fig. 3 (a), but further reduces the voltage swing at the output port to $270 \mathrm{mV}_{\mathrm{pp}}$ for our experiment. For the optical signal generation experiment, the FPGA outputs are directly connected to microwave probes that contact the modulator's GSG transmission lines. A bias voltage is applied via bias-tee to adjust the operation point.

\section{A. Signal Generation}

To characterize the performance of the transmitter, OOK and BPSK signals are generated and recorded for offline signal analysis. The experimental results are summarized in Fig. 3 (b) and (c). OOK signals are obtained by biasing the SOH MZM at the quadrature point. A real-time oscilloscope records the output current of a fast pin photodiode delivered to a $50 \Omega$ impedance. We use Matlab for offline signal analysis. No post-equalization is employed. Eye diagrams for the electrical drive signal and the optical signal as received are depicted in Fig. 3 (a) and (b), respectively. We do not measure any errors in approximately 390.000 recorded bits. The extracted quality-factor $\mathrm{Q}=6.7$ for the transmission indicates error-free operation $\left(B E R \approx 10^{-11}\right.$ ). Comparison of both eye diagrams in Fig. 3 (a) and (b) shows that bandwidth limitations are mainly due to the GTX module and not due to the $\mathrm{SOH}$ device. This is in agreement with an independent characterization of the SOH MZM, which exhibited a modulation bandwidth of approximately $15 \mathrm{GHz}$ - well above the bandwidth of the GTX modules. Hence, without further bandwidth degradation, it would be possible to improve signal quality by increasing the length of the phase shifters, thereby improving modulation depth at the expense of device bandwidth. Alternatively, when keeping the device geometry, this modulator could be used at higher symbol rates if supported by the electronic driver.

With the same drive signals, but operating the modulator in the null point, BPSK signaling is achieved. We use an optical 
(a)

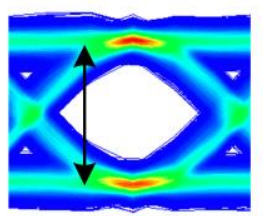

$0.28 \mathrm{~V}$

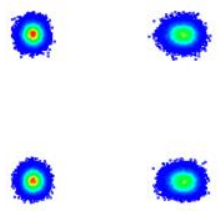

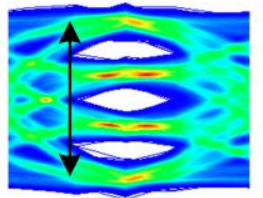

$0.41 \mathrm{~V}$

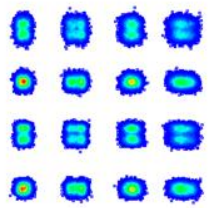

$6.7 \%$ (b)

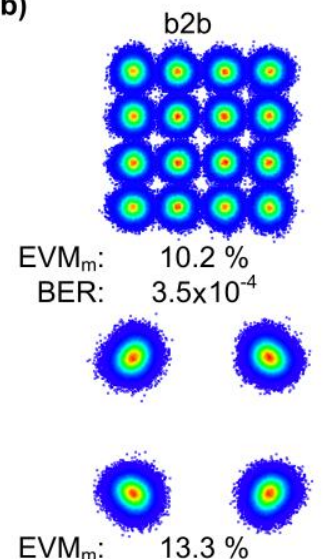

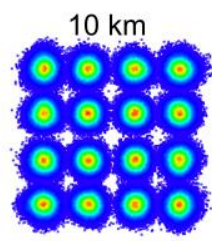

$10.3 \%$

$3.7 \times 10^{-4}$
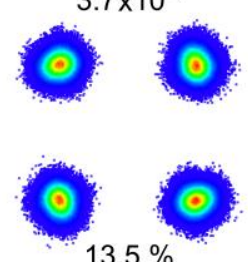

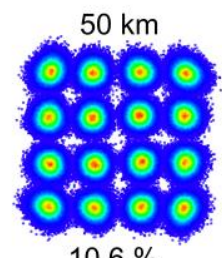

$10.6 \%$

$6.7 \times 10^{-4}$
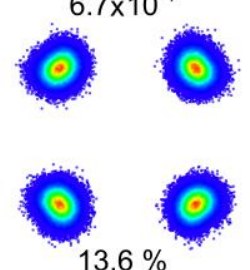

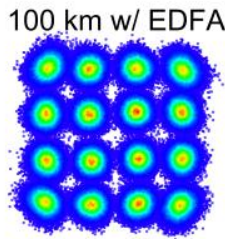

$11.1 \%$

$1.3 \times 10^{-3}$
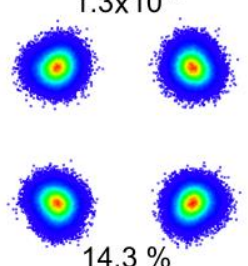

Fig. 6. (a) Electrical drive signals from FPGA GTH ports: Eye diagrams (top) for 2- (left) and 4-level (right) drive signals for QPSK and 16QAM. Output voltages of the drive signals for QPSK and 16QAM amount to $280 \mathrm{mV}_{\mathrm{pp}}$ and $410 \mathrm{mV}_{\mathrm{pp}}$, respectively. EVM values of the constellation diagrams (bottom) obtained after complex superposition of drive signals are measured to be $8.9 \%$ and $6.7 \%$ for QPSK and 16QAM, respectively after applying equalization at the receiver. (b) Optical constellation diagrams of optical 16QAM (top) and QPSK (bottom) signals generated (b2b) and transmitted over fiber spans of $10 \mathrm{~km}$, $50 \mathrm{~km}$ and $100 \mathrm{~km}$ length. An erbium-doped fiber amplifier (EDFA) at the receiver side was only used for a transmission distance of $100 \mathrm{~km}$. The influence of transmission distance on signal quality is small: For QPSK signals, the $\mathrm{EVM}_{\mathrm{m}}$ of $14.3 \%$ after $100 \mathrm{~km}$ still suggests BER values well below $10^{-9}$. For $16 \mathrm{QAM}$, the measured BER increases from $3.5 \times 10^{-4}$ to $1.2 \times 10^{-3}$, which is still below the 3rd generation FEC limit with $7 \%$ overhead [28].

modulation analyzer (OMA) with an external ECL acting as local oscillator (LO) for coherent detection. Signal analysis includes digital polarization alignment, frequency and phase estimation, and post-equalization. As a measure for signal quality, we use the error-vector magnitude (EVM), which represents the mean deviation of the measured constellation points from their ideal position in the complex plane [27]. For our BPSK signaling experiment, we measure an EVM of $13.7 \%$, which can be related to an estimate BER [27] well below $10^{-9}$ provided that signal performance is limited by Gaussian noise. This is consistent with the bit-error analysis of the recorded data stream of approximately $620 \mathrm{kbit}$ in which no bit errors were measured. The corresponding constellation and eye diagram for the BPSK signal are shown in Fig. 3(c). Even without post-equalization, error-free transmission is observed: The EVM is $21.5 \%$ (estimating BER $<10^{-10}$ ), and no errors were found in the recordings. These experiments show for the first time that non-resonant silicon-based MZM can be driven directly by standard FPGA outputs without further amplification.

\section{B. Transmission Experiment}

In a second experiment, we demonstrate data transmission by using the FPGA board both as a date source and sink. We again transmit OOK signals and detect them by a high-speed photodiode, but now connect the photodiode output to a GTX receiver on the same board, Fig. 4, thus simulating an electrical end-to-end communication system with optical transmission interface. No additional electrical amplifiers are used at the transmitter or receiver side. EDFA and BPF are again used for loss compensation and elimination of ASE. Signal generation and reception is performed with the Chipscope software provided by Xilinx. We measure a realtime BER of $6.6 \times 10^{-6}$. This is significantly worse than in the transmission experiments with offline data processing, but still well above typical forward-error correction (FEC) thresholds of $4.5 \times 10^{-3}$ for a $7 \%$ overhead [28]. The lower reception quality is attributed to the single-ended operation of the differential GTX Rx input, which is fed by a high-speed photodiode having rather small peak-to-peak output voltages of $30 \mathrm{mV}_{\mathrm{pp}}$. This voltage is significantly smaller than the input voltage of $150 \mathrm{mV}$ that is specified for the GTX Rx. Nevertheless, this experiment shows the general viability of an electrical end-to-end communication system with an optical interface that does not require additional electrical components.

\section{DAC-LESS GENERATION OF ADVANCED MODULATION FORMATS USING SOH IQ MODULATORS}

In a second set of experiments, we increase the spectral efficiency of the optical signal by employing advanced modulation formats and thus increasing the number of encoded bits per symbol while maintaining the amplifier-less scheme for signal generation. While the generation of multilevel signals, for e.g. 16QAM, usually requires digital-toanalog converters (DAC), our scheme relies solely on the binary FPGA output signals and passive components. The setup is similar to the one used in the previous experiment, see Fig. 5. The SOH MZM modulator is replaced by a $1.5 \mathrm{~mm}$ long IQ modulator which is connected to the outputs of an FPGA's GTH lines operating at $13 \mathrm{GBd}$. As the device is longer than the MZM modulator, a lower $\pi$-voltage of $0.53 \mathrm{~V}$ is measured at DC. Considering the length difference, the values for the $\pi$-voltages of both modulators are in good agreement, leading to $\pi$-voltage-length products of less than $0.8 \mathrm{Vmm}$ for both devices.

For generating each of the $\mathrm{I}$ and $\mathrm{Q}$ drive signals in the 16QAM experiment, we combine two GTH lines by using a power coupler, Fig. 5 . The $6 \mathrm{~dB}$ power difference between the two signal components required for a bipolar 4-level signal is realized by reducing the output voltage of one output port via the FPGA software and internal circuitry. Fine adjustment of the binary output levels of the FPGA can be used to 
compensate the nonlinear transfer function of the MZM. Unlike the experiment described in Section III, no FIR filter is required for compensating the low-pass characteristic of the FPGA output since the GTH output ports of the VC7222 used here exhibit a higher cut-off frequency than the GTX ports of the VC707 used in the previous experiments. Without the use of the FIR, we profit from higher output voltages. The IQ modulator features an optical insertion loss of $26 \mathrm{~dB}$. Compared to the insertion loss of the MZM in Section III, the higher loss is due to longer phase-shifter sections and access waveguides. As before, device optimizations will lead to a significantly reduced insertion loss. Using the same assumptions as for the MZM, we expect that total on-chip insertion losses of less than $2.7 \mathrm{~dB}$ can be achieved for an IQ modulator with $1.5 \mathrm{~mm}$-long slot-waveguide phase shifters. This would translate to more than $7.4 \mathrm{dBm}$ of on-chip output power when limiting the optical power in the slot waveguides to $5 \mathrm{dBm}$. The EDFA can hence be omitted for data transmission. For packaged devices, fiber-to-fiber insertion losses of less than $6.3 \mathrm{~dB}$ are to be expected.

After amplification in a subsequent EDFA and transmission, the signal is fed to an optical modulation analyzer (OMA). The $90^{\circ}$ phase shift between I and Q component of the optical signal is adjusted by an optical pathlength imbalance between the two arms and choice of the appropriate wavelength. For simplicity, we set up a homodyne receiver by splitting the external cavity laser (ECL) output in a signal and a local oscillator (LO) path.

For the experiment, we use a Xilinx XC7VH580T FPGA on a VC7222 evaluation board. A PRBS of length $2^{31}-1$ is generated in the FPGA and sent to the GTH ports. At a symbol rate of $13 \mathrm{GBd}$, this leads to an aggregate data rate of $26 \mathrm{Gbit} / \mathrm{s}$ and $52 \mathrm{Gbit} / \mathrm{s}$ for the QPSK and 16QAM formats, respectively. In the case of QPSK signaling, peak-to-peak drive voltages of $0.28 \mathrm{~V}_{\mathrm{pp}}$ are measured for the two-level Iand $\mathrm{Q}$ signal before the bias-tees used to set the bias point of the modulator. For 16QAM, the peak-to-peak drive voltage of the four-level signals amounts to $0.41 \mathrm{~V}_{\mathrm{pp}}$. The bias-tees are connected to the microwave probes contacting the chip. Eye diagrams for the electrical drive signals are depicted in the top row of Fig. 6 (a). The quality of the 16QAM drive signals is impeded by the power couplers, which are specified to work in the range of 1-18 GHz only. To quantify signal quality of the drive signal, we plot the 2- and 4-level drive signals for I and $\mathrm{Q}$ in the complex plane and measure the error vector magnitude of the resulting constellation diagrams that are depicted in the bottom row of Fig. 6 (a). For measuring the EVM, we use the definition normalized to the maximum vector length of the constellation diagram denoted as $\mathrm{EVM}_{\mathrm{m}}$ [27]. Without using an equalizer at the receiver, $\mathrm{EVM}_{\mathrm{m}}$ values for the drive signals for QPSK and 16QAM are measured to be $13.6 \%$ and $8.0 \%$, respectively. Applying an equalizer decreases the $\mathrm{EVM}_{\mathrm{m}}$ values to $8.9 \%$ and $6.7 \%$, see Fig. 6 (a).

For the optical signal, additional to measuring EVM values, we also measure the BER. We record the signals with realtime oscilloscopes and perform offline signal analysis which includes post-equalization. Within our record length of approximately $1.6 \mathrm{Mbit}$, we do not measure any errors for the back-to-back (b2b) QPSK signal. This is in good agreement with the measured $\mathrm{EVM}_{\mathrm{m}}$ values of $13.3 \%$ which indicates a BER well below $10^{-9}$ [27]. For 16QAM b2b transmission, we measure a BER of $3.5 \times 10^{-4}$ and an $\mathrm{EVM}_{\mathrm{m}}$ of $10.2 \%$. The measured BER is in good agreement with the BER of $4 \times 10^{-4}$ which can be estimated from the EVM [27]. The corresponding constellation diagrams are depicted on the left hand side of Fig. 6 (b).

We then perform transmission experiments with fiber spans of $10 \mathrm{~km}, 50 \mathrm{~km}$ and $100 \mathrm{~km}$. The results of the signal quality analysis and the constellation diagrams for the $\mathrm{b} 2 \mathrm{~b}$ case and transmission are depicted in Fig. 6 (b). An additional optical amplifier at the receiver side is used only for transmission over $100 \mathrm{~km}$. For distances larger than $10 \mathrm{~km}$, digital compensation of chromatic dispersion is applied. The influence of transmission on the quality of QPSK signals is negligible, as the $\mathrm{EVM}_{\mathrm{m}}$ is still $14.3 \%$ for a transmission distance of $100 \mathrm{~km}$. No errors were found in the recordings and we estimate a BER $<10^{-9}$ from the $\mathrm{EVM}_{\mathrm{m}}$. Hence, we consider the signal error-free. For the transmission of 16QAM signals, there is no significant change in $\mathrm{EVM}_{\mathrm{m}}$ or BER for a $10 \mathrm{~km}$ fiber span. Even for transmission over up to $100 \mathrm{~km}$, the BER of the 16QAM signal is $1.3 \times 10^{-3}$, still below the limit of $4.5 \times 10^{-3}$ for 3rd generation hard-decision FEC coding with an overhead of $7 \%$ [28].

\section{RF ENERGY CONSUMPTION OF MODULATOR}

In our experiments, no additional drive circuitry is used to interface the electrical ports of the FPGA to the EO modulator. As a consequence, the modulator must be operated solely with the energy provided by the binary FPGA outputs, which amounts to approximately $100 \mathrm{fJ} / \mathrm{bit}$ for a data rate of $13 \mathrm{Gibt} / \mathrm{s}$, a peak-to-peak output voltage of $500 \mathrm{mV}_{\mathrm{pp}}$, and a $50 \Omega$ impedance. It is a unique feature of our $\mathrm{SOH}$ devices that they can be operated with a small fraction of this energy. For analysis of the modulator RF energy consumption, we assume that the terminated $50 \Omega$ transmission lines of each MZM can be modeled as a single $50 \Omega$ resistor in an equivalent-circuit representation. For an IQ modulator, we have to consider the power consumption of two MZMs. For 16QAM, the magnitude of the bipolar drive voltage of each MZM can have two levels that are related by a factor of $1 / 3$. Assuming equal distribution of all 16QAM symbols, both signal levels occur with equal probability. From this, the energy consumption can be calculated by dividing the power consumption by the bit rate [17],

$$
W_{\text {bit } 16 \mathrm{QAM}}=2\left[\frac{1}{2}\left(\frac{U_{\mathrm{d}}}{2}\right)^{2} \frac{1}{R}+\frac{1}{2}\left(\frac{1}{3} \frac{U_{\mathrm{d}}}{2}\right)^{2} \frac{1}{R}\right] \times \frac{1}{r_{16 \mathrm{QAM}}},
$$

where $U_{\mathrm{d}}$ is the peak-to-peak value of the drive voltage, $R=50 \Omega$ is the impedance of the termination and $\mathrm{r}_{16 \mathrm{QAM}}$ is the bit rate of the 16QAM signal. Inserting the values from our experiment into the equation, $U_{\mathrm{d}}=0.41 \mathrm{~V}$ and $r_{16 \mathrm{QAM}}=52 \mathrm{Gbit} / \mathrm{s}$, we find an energy consumption per bit of 
$W_{\text {bit, } 16 \mathrm{QAM}}=18 \mathrm{fJ} / \mathrm{bit}$. This is the lowest value that has been reported for 16QAM signaling. Note that this figure solely covers the electrical energy consumption and does not take into account the power needed to drive, e.g., the laser or the EDFA in our experiment. We expect that the EDFA can be omitted completely when using optimized devices with significantly reduced insertion loss.

\section{SUMMARY}

We report on a proof-of-concept experiment of DAC-less and amplifier-less generation and transmission of optical data signals by directly driving $\mathrm{SOH}$ modulators with the binary sub-Volt outputs of an FPGA. Using an SOH Mach-Zehnder modulator, we generate error-free OOK and BSPK signals at $10 \mathrm{Gbit} / \mathrm{s}$. We demonstrate the viability for end-to-end transmission by feeding the OOK signal to a photodiode which is connected to a GTX receiver port of the same FPGA. In a second set of experiments, we generate QPSK and 16QAM signals at $13 \mathrm{GBd}$ and transmit them over distances of up to $100 \mathrm{~km}$. QPSK remains error-free for all transmission distances; for 16QAM, the BER remains below the threshold for hard-decision forward-error correction with $7 \%$ overhead. The SOH IQ modulator is shown to be highly energy-efficient, enabling 16QAM signaling at an RF energy consumption of only $18 \mathrm{fJ} / \mathrm{bit}$ at a data rate of $52 \mathrm{Gbit} / \mathrm{s}$. To the best of our knowledge, this is the lowest value reported for 16QAM signaling in literature so far.

\section{REFERENCES}

[1] J. S. Orcutt, B. Moss, C. Sun, J. Leu, M. Georgas, J. Shainline, E. Zgraggen, H. Li, J. Sun, M. Weaver, S. Urošević, M. Popović, R. J. Ram, and V. Stojanović, "Open foundry platform for high-performance electronic-photonic integration.," Opt. Express, vol. 20, no. 11, pp. 12222-32, May 2012.

[2] P. J. Winzer and R.-J. Essiambre, "Advanced Modulation Formats for High-Capacity Optical Transport Networks," J. Light. Technol., vol. 24, no. 12, pp. 4711-4728, Dec. 2006.

[3] A. Konczykowska, J.-Y. Dupuy, and F. Jorge, "42 GBd 3-bit powerDAC for optical communications with advanced modulation formats in InP DHBT," Electron. Lett., vol. 47, no. 6, p. 389, 2011.

[4] D. Brunina, X. Zhu, K. Padmaraju, L. Chen, M. Lipson, and K. Bergman, "10-Gb/s WDM Optically-Connected Memory System using Silicon Microring Modulators," in 38th European Conference on Optical Communication (ECOC 2012), Amsterdam 2012, no. 1, p. Mo.2.A.5.

[5] M. Secondini, E. Forestieri, and F. Cavaliere, "Novel optical modulation scheme for 16-QAM format with quadrant differential encoding," in Photonics in Switching, 2009.

[6] G.-W. Lu, M. Sköld, P. Johannisson, J. Zhao, M. Sjödin, H. Sunnerud, M. Westlund, A. Ellis, and P. a Andrekson, "40-Gbaud 16-QAM transmitter using tandem IQ modulators with binary driving electronic signals.," Opt. Express, vol. 18, no. 22, pp. 23062-9, Oct. 2010.

[7] A. Chiba, T. Sakamoto, T. Kawanishi, and K. Higuma, "16-level quadrature amplitude modulation by monolithic quad-parallel MachZehnder optical modulator," Electron. Lett., vol. 46, no. 3, pp. 220-222, 2010.

[8] A. Shastri, M. Webster, G. Jeans, P. Metz, S. Sunder, B. Chattin, B. Dama, and K. Shastri, "Experimental Demonstration of Ultra-LowPower Single Polarization $56 \mathrm{~Gb} / \mathrm{s}$ QAM-16 Generation without DAC using CMOS Photonics," in 40th European Conference on Optical Communication (ECOC 2014), Cannes 2014, p. PD.2.5.

[9] S. Wolf, P. C. Schindler, G. Ronniger, M. Lauermann, R. Palmer, S. Koeber, D. Korn, W. Bogaerts, J. Leuthold, W. Freude, and C. Koos, "10 GBd SOH Modulator Directly Driven by an FPGA without

(c) () 2015 IEEE. Personal use of this material is permitted. Permission from IEEE must be obtained for all other uses, in any current or future media, including reprinting/republishing this material for advertising or promotional purposes, creating new collective works, for resale or redistribution to servers or lists, or reuse of any copyrighted component of this work in other works.
Electrical Amplification," in 40th European Conference on Optical Communication (ECOC 2014), Cannes 2014, p. Mo.4.5.4.

[10] S. Wolf, M. Lauermann, G. Ronniger, P. C. Schindler, R. Palmer, S. Koeber, W. Bogaerts, J. Leuthold, W. Freude, and C. Koos, "DAC-Less and Amplifier-Less Generation and Transmission of 16QAM Signals Using a Sub-Volt Silicon Photonic Modulator," in 40th European Conference on Optical Communication (ECOC 2014), Cannes 2014, p. PD.4.5.

[11] T. Baehr-Jones, M. Hochberg, G. Wang, R. Lawson, Y. Liao, P. A. Sullivan, L. Dalton, A. K.-Y. Jen, and A. Scherer, "Optical modulation and detection in slotted Silicon waveguides," Opt. Express, vol. 13, no. 14, p. 5216, 2005.

[12] R. Palmer, S. Koeber, D. L. Elder, M. Woessner, W. Heni, D. Korn, M. Lauermann, W. Bogaerts, L. Dalton, W. Freude, J. Leuthold, and C. Koos, "High-Speed, Low Drive-Voltage Silicon-Organic Hybrid Modulator Based on a Binary-Chromophore Electro-Optic Material," J. Light. Technol., vol. 32, no. 16, pp. 2726-2734, 2014.

[13] J. Leuthold, C. Koos, W. Freude, L. Alloatti, R. Palmer, D. Korn, J. Pfeifle, M. Lauermann, R. Dinu, S. Wehrli, M. Jazbinsek, P. Guenter, M. Waldow, T. Wahlbrink, J. Bolten, H. Kurz, M. Fournier, J.-M. Fedeli, H. Yu, and W. Bogaerts, "Silicon-Organic Hybrid ElectroOptical Devices," IEEE J. Sel. Top. Quantum Electron., vol. 19, no. 6, p. 3401413, Nov. 2013.

[14] M. Lauermann, R. Palmer, S. Koeber, P. C. Schindler, D. Korn, T. Wahlbrink, J. Bolten, M. Waldow, D. L. Elder, L. R. Dalton, J. Leuthold, W. Freude, and C. Koos, "16QAM Silicon-Organic Hybrid (SOH) Modulator Operating with $0.6 \mathrm{Vpp}$ and $19 \mathrm{fJ} / \mathrm{bit}$ at $112 \mathrm{Gbit} / \mathrm{s}$," in CLEO: Science and Innovations, 2014, vol. 2, p. SM2G.6.

[15] R. Palmer, S. Koeber, W. Heni, D. L. Elder, D. Korn, H. Yu, L. Alloatti, S. Koenig, P. C. Schindler, W. Bogaerts, M. Pantouvaki, G. Lepage, P. Verheyen, J. Van Campenhout, P. Absil, R. Baets, L. R. Dalton, W. Freude, J. Leuthold, and C. Koos, "High-speed silicon-organic hybrid (SOH) modulator with 1, $6 \mathrm{fJ} / \mathrm{bit}$ and $180 \mathrm{pm} / \mathrm{V}$ in-device nonlinearity," in 39th European Conference and Exhibition on Optical Communication (ECOC 2013), London 2013, p. We.3.B.3.

[16] S. Koeber, R. Palmer, M. Lauermann, W. Heni, D. L. Elder, D. Korn, M. Woessner, L. Alloatti, S. Koenig, P. C. Schindler, H. Yu, W. Bogaerts, L. R. Dalton, W. Freude, J. Leuthold, and C. Koos, "Femtojoule electrooptic modulation using a silicon-organic hybrid device," Accept. Publ. Light Sci. Appl., 2014.

[17] M. Lauermann, R. Palmer, S. Koeber, P. C. Schindler, D. Korn, T. Wahlbrink, J. Bolten, M. Waldow, D. L. Elder, L. R. Dalton, J. Leuthold, W. Freude, and C. Koos, "Low-power silicon-organic hybrid (SOH) modulators for advanced modulation formats," Opt. Express, vol. 22, no. 24, pp. 29927-29936, Nov. 2014.

[18] L. Alloatti, D. Korn, R. Palmer, D. Hillerkuss, J. Li, A. Barklund, R. Dinu, J. Wieland, M. Fournier, J. Fedeli, H. Yu, W. Bogaerts, P. Dumon, R. Baets, C. Koos, W. Freude, and J. Leuthold, "42.7 Gbit/s electrooptic modulator in silicon technology," Opt. Express, vol. 19, no. 12, pp. 11841-11851, 2011.

[19] L. Alloatti, M. Lauermann, C. Sürgers, C. Koos, W. Freude, and J. Leuthold, "Optical absorption in silicon layers in the presence of charge inversion/accumulation or ion implantation," Appl. Phys. Lett., vol. 103, p. 051104, 2013.

[20] L. Alloatti, R. Palmer, S. Diebold, K. P. Pahl, B. Chen, R. Dinu, M. Fournier, J.-M. Fedeli, T. Zwick, W. Freude, C. Koos, and J. Leuthold, "100 GHz silicon-organic hybrid modulator," Light Sci. Appl., vol. 3, no. 5, p. e173, May 2014.

[21] R. Ding, T. Baehr-Jones, W.-J. Kim, B. Boyko, R. Bojko, A. Spott, A. Pomerene, C. Hill, W. Reinhardt, and M. Hochberg, "Low-loss asymmetric strip-loaded slot waveguides in silicon-on-insulator," Appl. Phys. Lett., vol. 98, no. 23, p. 233303, 2011.

[22] R. Palmer, L. Alloatti, D. Korn, W. Heni, P. C. Schindler, J. Bolten, M. Karl, M. Waldow, T. Wahlbrink, W. Freude, C. Koos, and J. Leuthold, "Low-Loss Silicon Strip-to-Slot Mode Converters," IEEE Photonics J., vol. 5, no. 1, pp. 2200409-2200409, Feb. 2013.

[23] R. Palmer, L. Alloatti, D. Korn, P. C. Schindler, R. Schmogrow, W. Heni, S. Koenig, J. Bolten, T. Wahlbrink, M. Waldow, H. Yu, W. Bogaerts, P. Verheyen, G. Lepage, M. Pantouvaki, J. Van Campenhout, P. Absil, R. Dinu, W. Freude, C. Koos, and J. Leuthold, "SiliconOrganic Hybrid MZI Modulator Generating OOK, BPSK and 8-ASK Signals for Up to $84 \mathrm{Gbit} / \mathrm{s}$," IEEE Photonics J., vol. 5, no. 2, pp. 6600907-6600907, Apr. 2013.

[24] D. Vermeulen, S. Selvaraja, P. Verheyen, G. Lepage, W. Bogaerts, P. Absil, D. Van Thourhout, and G. Roelkens, "High-efficiency fiber-to- 
chip grating couplers realized using an advanced platform," vol. 18, no. 17, pp. 18278-18283, 2010.

[25] N. Lindenmann, G. Balthasar, D. Hillerkuss, R. Schmogrow, M. Jordan, J. Leuthold, W. Freude, and C. Koos, "Photonic wire bonding: a novel concept for chip-scale interconnects.," Opt. Express, vol. 20, no. 16, pp. 17667-77, Jul. 2012.

[26] P. Dong, W. Qian, S. Liao, H. Liang, C.-C. Kung, N.-N. Feng, R. Shafiiha, J. Fong, D. Feng, A. V Krishnamoorthy, and M. Asghari, "Low loss shallow-ridge silicon waveguides.," Opt. Express, vol. 18, no. 14, pp. 14474-9, Jul. 2010.
[27] R. Schmogrow, B. Nebendahl, M. Winter, A. Josten, D. Hillerkuss, S. Koenig, J. Meyer, M. Dreschmann, M. Huebner, C. Koos, W. Freude, and J. Leuthold, "Error vector magnitude as a performance measure for advanced modulation formats," Photonics Technol. Lett. IEEE, vol. 24, no. 1, pp. 61-63, 2011.

[28] F. Chang, K. Onohara, and T. Mizuochi, "Forward error correction for $100 \mathrm{G}$ transport networks," Commun. Mag. IEEE, vol. 48, no. 3, pp. 48$55,2010$. 